\title{
Mucoadhesive Drug Delivery Systems: A Review of Recent Developments
}

\author{
Sharaf Alawdi ${ }^{1}$, Ajay B. Solanki ${ }^{1}$ \\ ${ }^{1}$ Gujarat Technological University, India. \\ ${ }^{2}$ Gujarat Technological University, India.
}

\begin{tabular}{l} 
Article Info \\
\hline Article history: \\
Received:11 January 2020 \\
Revised:25 February 2021 \\
Accepted:26 February 2021 \\
\hline Keywords: \\
Bioadhesive, \\
Polymers, \\
Mucoadhesive, \\
Patches, \\
Film. \\
Paper Type : \\
Review Article \\
Corresponding Author: \\
Sharaf Alawdi \\
Email: \\
mohasha17@gmail.com
\end{tabular}

\section{Introduction}

Since the early 1980s, the term of mucoadhesion has earned considerable attention in pharmaceutical technology(Mamatha et al., 2012) when professor Joseph R. Robinson from Wisconsin University pioneered the term of mucoadhesion as a new approach to extend the residence time of drugs used on the ocular surface(Verma et al., , 2011). The adhesion can be described as the affinity of dissimilar surfaces or particles to cling to one another while cohesion is the tendency of similar or identical surfaces/particles to hang on to one another(Szmyd \& Wasilewski, 2018). The American Society of Testing and Materials has described the adhesion as the state in which two surfaces are tied together by interfacial forces, consisting of interlocking action, valence forces, or both(Berndt \& Lin, 1993). Bioadhesion refers to any bond created between natural and synthetic materials with a biological surface, used mainly to describe the adhesion between polymers and soft tissue(Favi et al., 2014).

Mucoadhesive drug delivery systems promote the dosage form's residence time at the absorption site. They facilitate a close interaction of the dosage form with the 
underlying absorption surface and hence improve the drug's therapeutic performance as well as controlled drug release(Hombach \& Bernkop-Schnürch, 2010). The formulation of sustain release dosage form can gradually release the active drug for an extended period, but this is not enough to sustain a therapeutic effect. They may be eliminated from the site of absorption before emptying the drug content. Instead of that, the mucoadhesive dosage form will help both the purposes of sustaining the release and the incidence of dosage form at the site of absorption(Boddupalli et al., 2010).

In current years, several mucoadhesive drug delivery systems have been developed for nasal, buccal, oral, vaginal, and rectal routes for both local and systemic effects(Edsman \& Hägerström, 2005; Sofi, Abdal-Hay, Ivanovski, Zhang, \& Sheikh, 2020). For example nasal mucoadhesive microspheres of lercanidipine has been prepared by to improve systemic bioavailability and antihypertensive activity(Beg et al., 2020), A tenolol-releasing buccal patches has been formulated to avoid the extensive drug first pass metabolism(Hasnain et al., 2020), tenofovir mucoadhesive vaginal tablets designed to prevent sexual transmission of HIV(Notario-Pérez et al., 2018), and rectal mucoadhesive hydrogel prepared to prolong duration ofaction and increased bioavailability of anti-inflammatory Tolmetin (Ramadan et al., 2018).

This review is an attempt to present the most recent development regarding Mucoadhesive Drug Delivery Systems. In addition, the aim is to highlight the factors that affect the mucoadhesive dosage forms. For this purpose, an extensive literature review was conducted. Relevant databases were searched through Google Scholar and PubMed, and the keywords entered were Mucoadhesive Drug Delivery Systems, mucoadhesion, mucoadhesive forms, and factors affecting mucoadhesion.

\section{Literature Review}

\section{Mucous Membranes}

Mucous membranes (mucosae) are the moistened surfaces lining various walls of body cavities such as the respiratory tract and gastrointestinal tract(Smart, 2005). The main functions of the mucous membrane are to protect against infectious agents such as fungi, bacteria, and viruses. To work as a barrier in tissue absorption of the drugs, impacts the drug's bioavailability, mucus has strong adhesion properties and firmly binds the surface of the epithelial cells as a continuous gel layer. It also makes a lubrication to keep the mucus membrane moist. Specific mucous membranes possess specialized functions. For example, the intestinal and gastric mucosae are involved with food digestion and absorption. The nasal and olfactory mucosae aid odors to break down in the nose so that their particles can be perceived, and these substances can be smelled. Mucosae are also found in the reproductive system such as the vagina; naturally occurring vaginal discharge is produced by the vaginal mucosa to self-clean and keep the vagina moist and so on (Bhalerao \& Shinde, 2013; Boddupalli et al., 2010; Harris \& Robinson, 1992; Shaikh et al., 2011a).

\section{Mucoadhesive Dosage Forms}

These dosage forms (figure 2) are usually used to deliver drugs to the epithelial surfaces such as buccal, sublingual, oral, eye, nasal cavity, vagina, lung etc. They contain 
mucoadhesive polymers (biodegradable) to increase retention on dynamic mucosal surfaces and control the drug release(Nafee et al., 2004; Singh \& Tibrewal, 2017; Smart, 2005).

Figure 1: Figure 1: Main Mucoadhesive Dosage Forms

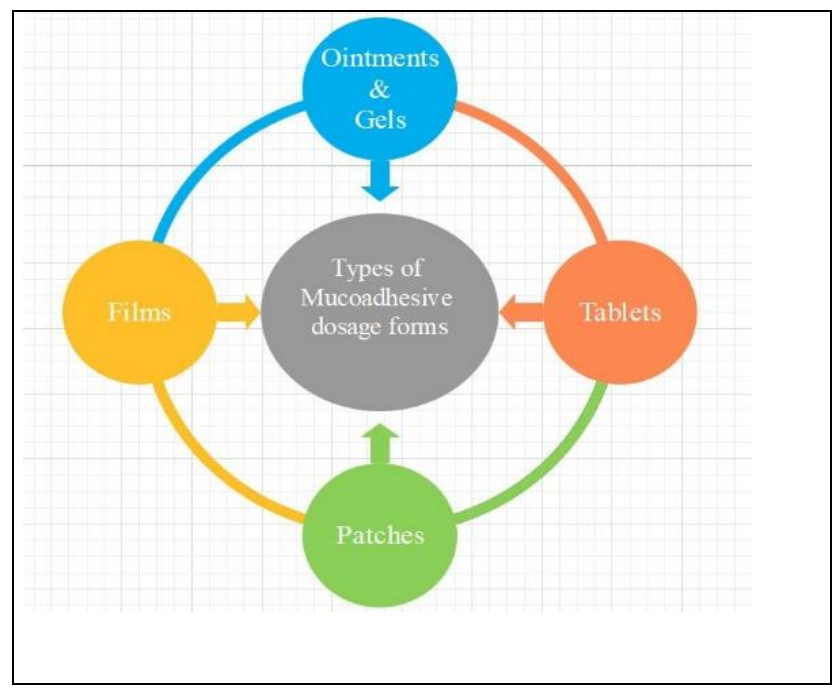

Source: Adapted from Nair et al. (2013)

\section{Mucoadhesive Tablets:}

Unlike traditional tablets, mucoadhesive tablets can be used on the buccal or sublingual or oral to allow fast onset of action and controlled drug delivery through increasing gastric retention time of the dosage forms(Abruzzo, Cerchiara, Bigucci, Gallucci, \& Luppi, 2015; Deshpande, Shah, Rhodes, \& Malick, 1997). They adhere to the mucosa and remain held in position till dissolution and/or release is complete. Mucoadhesive tablets provide efficient absorption and better bioavailability of the drugs because of a large surface to volume ratio and promotes a much closer connection with the mucus layer(Mathew, 2015). Mucoadhesive tablets are used widely because they release the drug for a prolonged period, reduce drug administration frequency, and improve patient compliance(Rajput et al., 2010). The significant drawback of mucoadhesive pills is their lack of physical flexibility, leading to poor patient compliance for long-term and repeated administration(Mathew, 2015).

\section{Gels and Ointments}

Semi-solid dosage forms, such as ointments and gels, benefit from easy distribution throughout the oral mucosa, vagina or eye(Chang et al., 2002). Thoughdrug dosing from semi-solid dosage forms may not be as reliable as from patches, tablets, or films, Low retention of the gels at the site of administration has been overwhelmed by using specific mucoadhesive polymers, such as sodium carboxy-methyl-cellulose, Carbopol, and xanthan gum, undergo a phase transition from liquid to semi-solid. This change magnifies the viscosity, which leads to a sustained and controlled drug release(Baloglu et al., 2009). 


\section{Films}

Mucoadhesive films (buccal or sublingual films) may be fancied over adhesive tablets in terms of flexibility and comfort. Besides, they can bypass the relatively short residence time of oral gels on the mucosa, which are simply washed away and transported by saliva. Furthermore, in the case of local delivery for oral diseases, the films further protect the wound surface, thus decreasing pain and treating the disease more effectively(Shaikh, Singh, Garland, Woolfson, \& Donnelly, 2011b). An excellent film should be elastic, flexible, and soft, yet adequately durable to withstand damage due to stress from mouth movements. It also needs superior mucoadhesive strength to be maintained in the mouth for the wanted duration of action(Nair et al., 2013).

\section{Patches}

Patches are laminates consisting of an impermeable waterproof backing layer, a drug comprising reservoir layer from which the drug is discharged in a controlled manner, and a mucoadhesive surface for mucosal connection(Koyi, 18/11/2013; Koyi \& Khan, 2013). Patch systems are similar to those used in transdermal drug delivery. Many approaches are applied to prepare adhesive patches, such as direct milling and solvent casting methods. In the solvent casting method, the intermediate sheet from which patches are punched is set up by casting the drug and polymer(s) solution onto a backing layer sheet and consequently leaving the solvent(s) to evaporate(Alhijjaj, Bouman, Wellner, Belton, \& Qi, 2015; Laffleur, 2014; Muhammad Umar Javaid, 2017; Srivastava \& Monga, 2015). Still, in the direct milling method, formulation constituents are homogeneously blended and compressed to the wanted thickness, and patches of predetermined size and shape are then cut or punched out(Kumar et al., 2014). An impermeable backing layer may also be employed to control the direction of drug release, prevent drug loss, and minimize deformation and disintegration of the device during the application period( Javaid, 2017).

\section{Need of Mucoadhesive Dosage Forms:}

Previous literature indicates a need for different forms of mucoadhesive dosage. Several studies present numerous advantages for mucoadhesive dosage forms. The following points present the results of some studies.

1- Mucoadhesive dosage forms give various advantages over other oral dosage forms and oral controlled release systems by prolonging the drug's residence time in the gastrointestinal tract or other mucous membranes(Netsomboon \& BernkopSchnürch, 2016).

2- Localization and targeting of the dosage form at a specific site(Boddupalli et al., 2010).

3- Provide close contact between dosage form and the site of application or absorption(Roy \& Prabhakar, 2010).

4- Application of dosage forms to mucosal surfaces could benefit drug molecules not amenable to the oral route, for instance those that undergo extensive first-pass metabolism or acid degradation(Boddupalli et al., 2010).

5- To improve the therapeutic achievement of drug and Reduction in the fluctuation 
of the steady-state plasma level(Netsomboon \& Bernkop-Schnürch, 2016).

6- It can provide controlled drug release(preferably unidirectional release) (Boddupalli et al., 2010).

7- To avoid first pass metabolism and GIT degradation by mucoadhesive routes such as buccal, sublingual, ocular, nasal, vaginal routes.

Disadvantages of Mucoadhesive Dosage Forms (Madhavi et al., 2013; Rossi et al.,, 2005; Salamat-Miller et al.,, 2005; Verma et al., 2011 ):

1- Oral mucoadhesive dosage form that contains ulcerative or irritant drugs due to their prolonged contact may cause local ulcerous effects.

2- It is not suitable for high doses and the involuntarily swallowing of buccal patches or films may cause choking.

\section{Polymers Used in Mucoadhesive Drug Delivery System:}

Mucoadhesive polymers are water-soluble and water insoluble polymers that adhere to the mucin-epithelial surface to provide prolonged contact time at the site of absorption. Ideal mucoadhesive polymer should be nontoxic, non-irritant and non-absorbable, adhere quickly to mucosa, inexpensive, stable during the shelf life, and build a strong non-covalent bonds with the mucin-epithelial cell surfaces(Bernkop-Schnürch, 2005; Mythri, Kavitha, Kumar, \& Singh, 2011).

Table 1: Classification of mucoadhesive polymers(Brannigan \& Khutoryanskiy, 2019; Grabovac, Guggi, \& Bernkop-Schnürch, 2005; Wang \& Ye, 2010)

\begin{tabular}{|c|c|c|}
\hline Criteria & Category & Example \\
\hline \multirow[t]{2}{*}{ Source } & Natural & $\begin{array}{l}\text { Chitosan, Agarose, Hyaluronic acid, Gelatin, } \\
\text { Pectin, Tragacanth,Gums (guar, Karaya, Xanthan, } \\
\text { etc.) }\end{array}$ \\
\hline & Synthetic & $\begin{array}{l}\text { Sodium Carboxy Methyl Cellulose } \\
\text { methyl cellulose, Carboxy Methyl }\end{array}$ \\
\hline \multirow[t]{2}{*}{ solubility } & Water soluble & $\begin{array}{l}\text { Carbopol, poly (vinyl pyrrolidone), Sodium } \\
\text { carboxy methyl cellulose, polyacrylic acid, Pectin, } \\
\text { xanthan gumandSodium alginate. }\end{array}$ \\
\hline & Water insoluble & Chitosan, polycarbophil, ethyl cellulose \\
\hline \multirow[t]{3}{*}{ charge } & Anionic & $\begin{array}{l}\text { Chitosan, Sodium alginate, Carbopol, Xanthan } \\
\text { gum, Pectin, polycarbophil. }\end{array}$ \\
\hline & Cationic & $\begin{array}{l}\text { Chitosan, Amino dextran, } \\
\text { dimethylaminoethyl dextran }\end{array}$ \\
\hline & $\begin{array}{l}\text { Nonionic } \\
\text { (neutral) }\end{array}$ & $\begin{array}{l}\text { Poly vinyl alcohol, Hydroxyl propyl cellulose, } \\
\text { Hydroxy ethyl starch, poly vinyl pyrrolidone }\end{array}$ \\
\hline
\end{tabular}

Source: Authors 


\section{Use of Polymers in Newer MDDS:}

Routinely mucoadhesive polymers are utilized to design and develop distinctive drug delivery platforms, for example, gel, microspheres, beads, patch, etc. via oral, buccal, nasal, vaginal routes, etc. Examples of such systems are widely available under the current scientific domain. Other than these, some relatively newer delivery systems are also recorded and becoming fashionable among researchers (Azzam, 2012; Bappaditya Chatterjee, Nursazreen Amalina, Pinaki Sengupta, \& Uttam Kumar Mandal, 2017; Khutoryanskiy, 2011). Mucoadhesive nano-carriers are the most prevalent. As a recent example, Luo, Teng, $\mathrm{Li}$, and Wang designed and developed a solid-lipid nanoparticle coated with chitosan. Because of chitosan, the nanoparticle revealed improved mucoadhesive property following oral delivery(Luo, Teng, Li, \& Wang, 2015). In another new work, Oh and Borros (2016) investigated mucoadhesion, and mucous permeability of thiolated chitosan made nanoparticle and observed significant results concerning both properties(Oh \& Borrós, 2016). A nanogel was formulated using conjugated chitosan and carboxymethyl chitosan by electrostatic interaction. It was shown that the nanogel can adhere to the intestinal mucosa for a prolonged duration of time, which in turn results in better drug action in colorectal cancer(Bappaditya Chatterjee, Nursazreen Amalina, Pinaki Sengupta, \& Uttam Kumar Mandal, 2017). According to Sosnik et al. (2014), the mucoadhesive polymers for the non-parenteral delivery system have been described. As per the authors, all three types of polymers such as natural (pectin, alginate, chitosan, hyaluronic acid, etc.), synthetics (acrylic acid derivatives), and semi-synthetic (cellulose derivatives) are used for the nanoparticulate mucoadhesive delivery system(Sosnik, das Neves, \& Sarmento, 2014). In another non-conventional mucoadhesive approach, Horvat et al. (2015) developed an in-situ ocular mucoadhesive gel, where the authors have used thiolated poly aspartic acid, which can also be considered as a nonconventional mucoadhesive carrier. The thio groups of poly aspartic acid can form disulphide linkage with mucin glycoprotein and cause mucoadhesion. The delivery system showed promising prolonged drug release up to $24 \mathrm{hrs}$. Apart from these, few more research types on newer types of MDDS are highly prevalent in the pharmaceutical or bio-engineering field, for example, delivery of insulin via intestinal device or floating bioadhesivemultiparticulate delivery via hollow structure(Horvát et al., 2015). Another exciting research was done by Abu-Huwaij(Abu-Huwaij, Hamed, Daoud, \& Alkilani, 2019)et al (2019) developed nanoemulsion-based buccal patches of valsartan. The prepared unidirectional buccal patches provided a maximum drug release within specified mucoadhesion period and this indicates a potential alternative drug delivery system for systemic delivery of carbamazepine(AbuHuwaij et al., 2019).

\section{Mechanism of Mucoadhesion}

The mechanism by which a mucoadhesive bond is created depends largely on numerous factors such as the mucoadhesive material, the formulation, the mucous membrane nature, the bond's subsequent environment, and the way of attachment. The chemical bonds responsible for the mucoadhesion are strong bonds that support the formulation to stick to the mucosa as ionic bonds and covalent bond and weak bonds like hydrogen bonds, Van-der Waals bonds. In addition to other hydrophobic bonds, understanding of these mechanisms in 
each incidence helps in the development of new, improved drug delivery systems (Alexander et al., 2011; Carvalho et al., 2010a, 2010b; Smart, 2005; Sudheer, 2018).

Theories of Mucoadhesion:

1- Electronic theory(Vasir, Tambwekar, \& Garg, 2003): It is based on the suggestion that both mucoadhesive and biological materials possess opposing electrical charges. Thus, when both materials come into contact, they transfer electrons leading to the building of a double electronic layer at the interface, where the attractive forces within this electronic double layer determine the mucoadhesive strength.

2- Adsorption theory(Woertz, Preis, Breitkreutz, \& Kleinebudde, 2013): In this theory, the mucoadhesive device holds fast to the mucous after contact because of surface force acting between the atoms in both surfaces. From this force, a secondary chemical interaction, for example, in van der Waals and hydrogen bonds, electrostatic fascination, or hydrophobic interactions rise.

3- Wetting theory(Rojewska et al., 2017): The wetting theory applies to liquid systems which is the affinity of a liquid to maintain contact in the surface. This affinity can be found by using measuring methods such as the contact angle. The common rule states that the lower the contact angle, the more significant the affinity (Figure 3). For sufficient spreading and completely wetting liquid, contact angle must be zero or close to zero.

The difference between the surface energies $\gamma \mathrm{B}$ and $\gamma \mathrm{A}$ and the interfacial energy $\gamma \mathrm{AB}$, as indicated in equation

(Equation 1) $\quad S_{A B}=\gamma_{B}-\gamma_{A}-\gamma_{A B}$

The greater the individual surface energy of mucus and device concerning the interfacial energy, the greater the adhesion work, WA, i.e., the greater the energy needed to separate the two phases

(Equation 2) $W_{A}=\gamma_{A}+\gamma_{B}-\gamma_{A B}$

4- Diffusion theory: Dissemination theory portrays the interpenetration of both polymer and mucin chains to an adequate depth to make a semi-permanent adhesive bond (figure 5). It is accepted that the adhesion force increments with the level of penetration of the polymer chains. This penetration rate relies upon the diffusion coefficient, flexibility, and nature of the mucoadhesive chains, mobility, and contact time. As indicated by the literature, the depth of interpenetration needed to create an effective bioadhesive bond lies in the range 0.2-0.5 $\mu \mathrm{m}$. This interpenetration depth of polymer and mucin chains can be assessed by the contact time, and $\mathrm{Db}$ is the diffusion coefficient of the mucoadhesive material in the mucus.

$$
l=\left(t D_{b}\right)^{1 / 2} \quad(\text { Equation } 3)
$$

The adhesion strength for a polymer is reached when the penetration depth is around proportional to the polymer chain size. For diffusion to happen, the components included must have great mutual solubility, that is, both the bioadhesive and the 
mucous have comparative chemical structures - the more the structural similarity, the better the mucoadhesive bond.

5- Mechanical theory(Yadav, Gupta, Kumar, Yadav, \& Kumar, 2010): This theory believes that adhesion is be owing to the filling of the irregularities on a rough surface by the used mucoadhesive liquid. Besides, such irregularity rises the interfacial area existing for the interactions, thus aiding scattering energy and can be considered the most important phenomenon of the process. It is far-fetched that the mucoadhesion process is the same for all cases, and therefore it cannot be described by a solitary theory. All theories are relevant to recognize the critical process variables. The mechanisms regulating mucoadhesion are also influenced by the intrinsic properties of the formulation and by the environment in which it is applied. Inherent factors of the polymer are linked to its molecular weight, concentration, and chain flexibility.

6- Fracture theory:Maybe this is the most-utilized theory in studies on the mechanical estimation of mucoadhesion.it differs the previous theories in it relates the adhesive strength to the forces required for detatchement of the two involved surfaces after adhesion. It analyses the force needed to separate two surfaces after adhesion is established. This force, $S_{\mathrm{m}}$, is frequently determined in tests of resistance to rupture by the ratio of the maximal detachment force, Fm, and the total surface area, $\mathrm{A}_{0}$, involved in the adhesive interaction.

$$
s_{m}=\frac{F_{m}}{A_{0}}
$$

(Equation 4)

In a single component uniform system, the fracture force, SJ, which is equivalent to the maximal rupture tensile strength, $S_{\mathrm{m}}$, is proportional to the fracture energy $\left(\mathrm{G}_{\mathrm{C}}\right)$ for Young's module (E) and to the critical breaking length (c) for the fracture site, as described in the following equation.

$s_{f} \sim\left(\frac{g_{c} E}{c}\right)^{1 / 2}$

\section{(Equation 5)}

Fracture energy $\left(\mathrm{G}_{\mathrm{C}}\right)$ can be obtained from the reversible adhesion work, $\mathrm{W}_{\mathrm{r}}$ (energy required to produce new fractured surfaces), and the irreversible adhesion work, Wi (work of plastic deformation provoked by the removal of a proof tip until the disruption of the adhesive bond), and both values are expressed as units of fracture surface (Af).

$g_{c}=W_{r}+W_{i}$

(Equation 6)

The elastic module of the system (E) is related to the stress (s) and to the shear (e) by Hooke's law:

$E=\left[\frac{\sigma}{\varepsilon}\right]_{\varepsilon \rightarrow 0}=\left[\frac{F / A_{0}}{\Delta l / l_{0}}\right]_{\Delta l \rightarrow o}($ Equation 7)

In equation 7 , the stress is the ratio between force (F) and area (A0), and shear is given by the ratio between the variety of system thickness (Dl) and the original thickness (1 0). A criticism of this analysis is that the system under investigation must 
have known physical dimensions and ought to be constituted by a single and uniform material. For this situation, the equation ought to be expanded to oblige elastic dimensions and modules for each component. Besides, it must be considered that a failure of adhesion will happen at the bioadhesive interface. Nonetheless, it has been demonstrated that the rupture seldom occurs at the surface, however close to it or at the most vulnerable point, which can be simply the interface itself, the mucus layer, or the hydrated region of the mucus, as represented in (Figure 6).Since the fracture theory is concerned distinctly with the force required to isolate the parts, it does not consider the interpenetration or diffusion of polymer chains. Therefore, it is proper for use in the calculations for rigid or semi-rigid bioadhesive materials, in which the polymer chains do not penetrate the mucus layer.

\section{Factors Affecting Mucoadhesion:}

In this review, certain factors affecting mucoadhesion are identified. The factors are either polymer-based, environmental or physiological. A brief account of these factors is presented and discussed below.

\section{A- Polymer based factors:}

1- Molecular weight: Many studies have revealed that the ideal molecular weight for better bio-adhesion, relies on the used bioadhesive polymer type. For example, polyethylene glycol (PEG), with a molecular weight of 20,000, possesses little adhesive strength, whereas PEG with a molecular weight of 200,000 get enhanced, and a PEG with 400,000 has superior adhesive properties. In linear polymers the bioadhesiveness increases with increasing of molecular weight. This fact implies two things: (a) interpenetration is more significant for polymers with lower molecular weight, and (b) entanglement is essential for higher molecular weight polymers.Nonlinear structure adhesiveness, follows a pretty different trend. For example, dextran adhesive strength, with molecular weight of 19,500,000, is similar to that of PEG, with a molecular weight 200,000 . The reason of similarity may be that the dextran helical conformation that may shield many groups responsible of adhesion, unlike the PEG conformation(Patel, Patel, \& Chaudhry, 2011).

2- Flexibility:

Bio-adhesion begins when the polymer chains diffuse into the interfacial region. Thepolymer flexibility is required to achieve the desired entanglement with the mucus.Generally, polymers viscosities and diffusion coefficients can have the main effect on the polymer flexibility and mobility, where higher flexibility of a polymer leads to greater diffusion into the mucus network(Okutan, Terzi, \& Altay, 2014; Stastna, Zanzotto, \& Vacin, 2003).

3- Hydrogen bonding capacity: it is confirmed that a good hydrogen bonding is important in mucoadhesion and the polymer flexibility is important to improve this capacity(Lee, Park, \& Robinson, 2000; Park \& Robinson, 1987).

4- Cross linking capacity: increasing of cross-linking density will decrease the water diffusion into the polymer network which cause an insufficient swelling of the 
polymer and hence, the rate of interpenetration between the polymer and mucin will decrease leading to decrease the mucoadhesion(Tiwary \& Rana, 2010).

5- Charge: Nonionic polymers have smaller degree of adhesion compared to anionic polymers. Also, some cationic polymers such as chitosan show superior adhesion especially in slight alkaline or neutral(Leung \& Robinson, 1990).

6- Polymer concentration: low concentration may lead to unstable interaction between polymer and mucin, in general more concentrated polymer would cause longer penetrating chain length and better mucoadhesion. However, there is a critical concentration for each polymer above which the polymer chain penetration may be reduced(Yadav et al., 2010).

7- Hydration (swelling): the polymer swelling depends on the ionic strength, polymer concentration, and cross-linking density, as well as the presence of water. A critical degree of hydration of polymer exists where the optimum swelling and mucoadhesion occurs. Overhydration leads to wet slippery mucilage formation that prevent adhesion(Geraghty, Attwood, Collett, Sharma, \& Dandiker, 1997).

\section{B- Environmental and physiological factors affecting on mucoadhesion:}

1- $\mathrm{pH}$ : the site of administration $\mathrm{pH}$ influences the charge on the mucus surface as well as some ionizable polymers. Medium $\mathrm{pH}$ affects the degree of hydration of some polymers(e.g. polyacrylic acid)(Ende \& Peppas, 1996).

2- Initial contact time: the contact time of bioadhesive polymer and mucus layer determines the swelling extent interpenetration of polymer chains. Moreover, the strength of bioadhesive increases as the initial contact time increases(Vasir et al., 2003).

3- Mucin turn over: how long the bioadhesive remains at the administration site depends on solubility of polymer in water and the associated rate of mucin turnover(Roy \& Prabhakar, 2010).

4- Diseased state: in some disease states (e.g., in Dry Mouth Syndrome), the secretion of mucus from the mucus membrane gets decreased. Thus, the amount of mucus available is not sufficient to interact with bioadhesive polymer at the site of attachment leading to improper moistening and swelling of polymerand hence decreasing of mucoadhesive strength of mucoadhesive dosage forms(R. Singh, Sharma, \& Garg, 2017).

\section{Conclusion}

This study aimed at presenting a review of the development in mucoadhesion drug delivery systems. The review covered the mucoadhesive concepts, polymers used, theories and mechanisms of mucoadhesion, and factors affecting the mucoadhesive dosage forms. Relevant databases were searched through Google scholar and PubMed. Based on the literature available, it is identified that the majority of studies suggest mucoadhesive drug delivery systems as the best alternative approaches for the traditional dosage forms to enhance bioavailability of poorly soluble drugs and to avoid GIT degradation and first pass metabolism of some drugs. 


\section{Conflict of Interest}

We have no competing interests.

\section{Funding:}

The authors received no funding for conducting this study.

\section{References}

Abruzzo, A., Cerchiara, T., Bigucci, F., Gallucci, M. C., \& Luppi, B. (2015). Mucoadhesive buccal tablets based on chitosan/gelatin microparticles for delivery of propranolol hydrochloride. Journal of pharmaceutical sciences, 104(12), 4365-4372.

Abu-Huwaij, R., Hamed, R., Daoud, E., \& Alkilani, A. Z. J. A. P. P. (2019). Development and In Vitro Characterization of Nanoemulsion-Based Buccal Patches Of Valsartan. Acta Poloniae Pharmaceutica, 76(2), 313-321.

Alexander, A., Ajazuddin, S., Tripathi, D., Verma, T., Mayura, J., \& Patel, B. (2011). Mechanism responsible for mucoadhesion of mucoadhesive drug delivery system: a review. J International journal of applied biology pharmaceutical technology, 2(1), 434-445.

Alhijjaj, M., Bouman, J., Wellner, N., Belton, P., \& Qi, S. (2015). Creating drug solubilization compartments via phase separation in multicomponent buccal patches prepared by direct hot melt extrusion-injection molding. Molecular pharmaceutics, 12(12), 4349-4362.

Azzam, W. J. J. o. a. p. s. (2012). Reduction of the shrinkage-swelling potential with polymer nanocomposite stabilization. Journal of Applied Polymer Science, 123(1), 299-306.

Baloglu, E., Bernkop-Schnürch, A., Karavana, S. Y., \& Senyigit, Z. A. (2009). Strategies to prolong the intravaginal residence time of drug delivery systems. Journal of Pharmacy and Pharmaceutical Sciences, 12(3), 312-336.

Beg, S., Rahman, M., Panda, S. K., Alharbi, K. S., Alruwaili, N. K., Singh, P. K., . . Singh, B. (2020). Nasal mucoadhesive microspheres of lercanidipine with improved systemic bioavailability and antihypertensive activity. Journal of Pharmaceutical Innovation, $1-10$.

Berndt, C., \& Lin, C. (1993). Measurement of adhesion for thermally sprayed materials. Journal of Adhesion Science, 7(12), 1235-1264.

Bernkop-Schnürch, A. (2005). Thiomers: a new generation of mucoadhesive polymers. $J$ Advanced drug delivery reviews, 57(11), 1569-1582.

Bhalerao, M., \& Shinde, M. (2013). Novel Approaches for Mucoadhesive Gel-A Review. J Asian Journal of Pharmaceutical Research and Development, 156-170.

Boddupalli, B. M., Mohammed, Z. N., Nath, R. A., \& Banji, D. (2010). Mucoadhesive drug delivery system: An overview. Journal of advanced pharmaceutical technology and research, 1(4), 381.

Brannigan, R. P., \& Khutoryanskiy, V. V. (2019). Progress and current trends in the synthesis of novel polymers with enhanced mucoadhesive properties. $J$ Macromolecular bioscience, 19(10), 1900194.

Carvalho, F. C., Bruschi, M. L., Evangelista, R. C., \& Gremião, M. P. D. J. B. J. o. P. S. (2010a). Mucoadhesive drug delivery systems. 46(1), 1-17. 
Carvalho, F. C., Bruschi, M. L., Evangelista, R. C., \& Gremião, M. P. D. J. B. J. o. P. S. (2010b). Mucoadhesive drug delivery systems. J Brazilian Journal of Pharmaceutical Sciences, 46(1), 1-17.

Chang, J. Y., Oh, Y.-K., Choi, H.-g., Kim, Y. B., \& Kim, C.-K. (2002). Rheological evaluation of thermosensitive and mucoadhesive vaginal gels in physiological conditions. International journal of pharmaceutics, 241(1), 155-163.

Chatterjee, B., Amalina, N., Sengupta, P., \& Mandal, U. K. (2017). Mucoadhesive polymers and their mode of action: A recent update. Journal of Applied Pharmaceutical Science, 7(05), 195-203.

Chatterjee, B., Amalina, N., Sengupta, P., \& Mandal, U. K. (2017). Mucoadhesive polymers and their mode of action: A recent update. J Journal of Applied Pharmaceutical Science, 7(5), 195-203.

Deshpande, A. A., Shah, N. H., Rhodes, C. T., \& Malick, W. (1997). Development of a novel controlled-release system for gastric retention. J Pharmaceutical research, 14(6), 815-819.

Edsman, K., \& Hägerström, H. (2005). Pharmaceutical applications of mucoadhesion for the non-oral routes. Journal of pharmacy and pharmacology, 57(1), 3-22.

Ende, M. T. A., \& Peppas, N. A. (1996). Transport of ionizable drugs and proteins in crosslinked poly (acrylic acid) and poly (acrylic acid-co-2-hydroxyethyl methacrylate) hydrogels. I. Polymer characterization. Journal of applied polymer science, 59(4), 673-685.

Favi, P. M., Yi, S., Lenaghan, S. C., Xia, L., \& Zhang, M. (2014). Inspiration from the natural world: from bio-adhesives to bio-inspired adhesives. Journal of Adhesion Science and Technology, 28(3-4), 290-319.

Geraghty, P., Attwood, D., Collett, J., Sharma, H., \& Dandiker, Y. (1997). An investigation of the parameters influencing the bioadhesive properties of Myverol 18-99/water gels. J Biomaterials, 18(1), 63-67.

Grabovac, V., Guggi, D., \& Bernkop-Schnürch, A. (2005). Comparison of the mucoadhesive properties of various polymers. J Advanced drug delivery reviews, 57(11), 1713-1723.

Harris, D., \& Robinson, J. R. J. J. o. p. s. (1992). Drug delivery via the mucous membranes of the oral cavity. J Journal of pharmaceutical sciences, 81(1), 1-10.

Hasnain, M. S., Guru, P. R., Rishishwar, P., Ali, S., Ansari, M. T., \& Nayak, A. K. (2020). Atenolol-releasing buccal patches made of Dillenia indica L. fruit gum: preparation and ex vivo evaluations. J SN Applied Sciences, 2(1), 1-10.

Hombach, J., \& Bernkop-Schnürch, A. (2010). Mucoadhesive drug delivery systems. J Drug delivery, 251-266.

Horvát, G., Gyarmati, B., Berkó, S., Szabó-Révész, P., Szilágyi, B. Á., Szilágyi, A., . . . Rossi, S. (2015). Thiolated poly (aspartic acid) as potential in situ gelling, ocular mucoadhesive drug delivery system. European Journal of Pharmaceutical Sciences, $67,1-11$.

Khutoryanskiy, V. V. (2011). Advances in mucoadhesion and mucoadhesive polymers. $J$ Macromolecular bioscience, 11(6), 748-764.

Koyi, P. K. (18/11/2013). Formulation and in vitro Evaluation of Bilayered Buccal Patches of a Proton Pump Inhibitor. American Journal of Advanced Drug Delivery. 
Koyi, P. K., \& Khan, A. B. (2013). Buccal patches: a review. International Journal of Pharmaceutical Sciences Research, 4(1), 83.

Kumar, D. R., Kumar, K. V., Sarma, D., Kumar, K. S., Ahmad, S., \& Geethavani, M. (2014). Buccal Patches-A Review. J Research Journal of Pharmaceutical Dosage Forms, 6(3), 167-173.

Laffleur, F. (2014). Mucoadhesive polymers for buccal drug delivery. J Drug development industrial pharmacy, 40(5), 591-598.

Lee, J. W., Park, J. H., \& Robinson, J. R. (2000). Bioadhesive-based dosage forms: The next generation. Journal of pharmaceutical sciences, 89(7), 850-866.

Leung, S.-H. S., \& Robinson, J. R. (1990). Polymer structure features contributing to mucoadhesion. II. Journal of controlled release, 12(3), 187-194.

Luo, Y., Teng, Z., Li, Y., \& Wang, Q. (2015). Solid lipid nanoparticles for oral drug delivery: Chitosan coating improves stability, controlled delivery, mucoadhesion and cellular uptake. J Carbohydrate polymers, 122, 221-229.

Madhavi, B., Murthy, V. S., Rani, A., \& Kumar. (2013). Buccal film drug delivery system-an innovative and emerging technology. J Mol Pharm Org Process Res, 1(107), 2.

Mamatha, Y., Selvi, A., Prasanth, V., Sipai, M. A. B., \& Yadav, V. (2012). Buccal drug delivery: a technical approach. Journal of Drug Delivery and Therapeutics, 2(2).

Mathew, A. K. (2015). Oral local drug delivery: An overview. J Pharm Pharmacol Res, 3(1), $1-6$.

Muhammad Umar Javaid, S. S. (2017). Buccal Patches: An Advanced Route of Drug Dosage Delivery-A Review. international journal of pharmacy and pharmaceutical research, Vol. 10 (3): 206-216.

Mythri, G., Kavitha, K., Kumar, M. R., \& Singh, S. J. (2011). Novel mucoadhesive polymers-a review. Journal of Applied Pharmaceutical Science, 1(8), 37-42.

Nafee, N. A., Ismail, F. A., Boraie, N. A., \& Mortada, L. M. (2004). Mucoadhesive delivery systems. I. Evaluation of mucoadhesive polymers for buccal tablet formulation. $J$ Drug development industrial pharmacy, 30(9), 985-993.

Nair, A. B., Kumria, R., Harsha, S., Attimarad, M., Al-Dhubiab, B. E., \& Alhaider, I. A. (2013). In vitro techniques to evaluate buccal films. Journal of Controlled Release, 166(1), 10-21.

Netsomboon, K., \& Bernkop-Schnürch, A. (2016). Mucoadhesive vs. mucopenetrating particulate drug delivery. European Journal of Pharmaceutics

and Biopharmaceutics, 98, 76-89.

Notario-Pérez, F., Cazorla-Luna, R., Martín-Illana, A., Ruiz-Caro, R., Tamayo, A., Rubio, J., \& Veiga, M.-D. (2018). Optimization of tenofovir release from mucoadhesive vaginal tablets by polymer combination to prevent sexual transmission of HIV. $J$ Carbohydrate polymers, 179, 305-316.

Oh, S., \& Borrós, S. (2016). Mucoadhesion vs mucus permeability of thiolated chitosan polymers and their resulting nanoparticles using a quartz crystal microbalance with dissipation (QCM-D). J Colloids and Surfaces B: Biointerfaces, 147, 434-441.

Okutan, N., Terzi, P., \& Altay, F. (2014). Affecting parameters on electrospinning process and characterization of electrospun gelatin nanofibers. J Food Hydrocolloids, 39, 1926. 
Park, H., \& Robinson, J. R. (1987). Mechanisms of mucoadhesion of poly (acrylic acid) hydrogels. J Pharmaceutical research, 4(6), 457-464.

Patel, A. R., Patel, D. A., \& Chaudhry, S. V. (2011). Mucoadhesive buccal drug delivery system. International Journal of Pharmacy and Life Sciences, 2(6).

Rajput, G., Majmudar, F., Patel, J., Patel, K., Thakor, R., Patel, B., \& Rajgor, N. (2010). Stomach specific mucoadhesive tablets as controlled drug delivery system-A review work. J Int J Pharm Biol Res, 1, 30-41.

Ramadan, A. A., Elbakry, A. M., Esmaeil, A. H., \& Khaleel, S. A. (2018). Pharmaceutical and pharmacokinetic evaluation of novel rectal mucoadhesive hydrogels containing tolmetin sodium. Journal of pharmaceutical investigation, 48(6), 673-683.

Rojewska, M., Olejniczak-Rabinek, M., Bartkowiak, A., Snela, A., Prochaska, K., \& Lulek, J. (2017). The wettability and swelling of selected mucoadhesive polymers in simulated saliva and vaginal fluids. Colloids and Surfaces B: Biointerfaces, 156, 366374.

Rossi, S., Sandri, G., \& Caramella, C. M. (2005). Buccal drug delivery: a challenge already won? J Drug Discovery Today: Technologies, 2(1), 59-65.

Roy, S., \& Prabhakar, B. (2010). Bioadhesive polymeric platforms for transmucosal drug delivery systems-a review. Tropical Journal of Pharmaceutical Research, 9(1).

Salamat-Miller, N., Chittchang, M., \& Johnston, T. P. (2005). The use of mucoadhesive polymers in buccal drug delivery. J Advanced drug delivery reviews, 57(11), 16661691.

Shaikh, R., Singh, T. R. R., Garland, M. J., Woolfson, A. D., \& Donnelly, R. F. (2011a). Mucoadhesive drug delivery systems. J Journal of Pharmacy Bioallied Sciences, 3(1), 89.

Shaikh, R., Singh, T. R. R., Garland, M. J., Woolfson, A. D., \& Donnelly, R. F. (2011b). Mucoadhesive drug delivery systems. Journal of Pharmacy Bioallied Sciences, 3(1), 89.

Singh, P., \& Tibrewal, R. (2017). MUCOADHESIVE AND MICROSPHERE: A SHORT REVIEW. J International Journal of Medical

Biomedical Studies, 1(4).

Singh, R., Sharma, D., \& Garg, R. (2017). Review on mucoadhesive drug delivery system with special emphasis on buccal route: an important tool in designing of novel controlled drug delivery system for the effective delivery of pharmaceuticals. $J$ Dev Drugs, 6(01), 1-12.

Smart, J. D. J. A. d. d. r. (2005). The basics and underlying mechanisms of mucoadhesion. $J$ Advanced drug delivery reviews, 57(11), 1556-1568.

Sofi, H. S., Abdal-Hay, A., Ivanovski, S., Zhang, Y. S., \& Sheikh, F. A. (2020). Electrospun nanofibers for the delivery of active drugs through nasal, oral and vaginal mucosa: current status and future perspectives. J Materials Science and Engineering: C, 111, 110756.

Sosnik, A., das Neves, J., \& Sarmento, B. (2014). Mucoadhesive polymers in the design of nano-drug delivery systems for administration by non-parenteral routes: a review. $J$ Progress in Polymer Science, 39(12), 2030-2075.

Srivastava, N., \& Monga, M. G. (2015). Current Status of Buccal Drug Delivery System: a 
Review. J Journal of Drug Delivery Therapeutics, 5(1), 34-40.

Stastna, J., Zanzotto, L., \& Vacin, O. (2003). Viscosity function in polymer-modified asphalts. Journal of colloid and interface science, 259(1), 200-207.

Sudheer, P. J. J. o. P. R. (2018). Mucoadhesive polymers: A review. J Journal of Pharmaceutical Research, 17(1), 47-55.

Szmyd, M., Paliga, D., \& Wasilewski, A. (2018). Influence of the surface condition on the adhesion of composite materials to enamel. J Physics for Economy, 2, 43-54.

Tiwary, A. K., \& Rana, V. (2010). Cross-linked chitosan films: effect of cross-linking density on swelling parameters. Pak J Pharm Sci, 23(4), 443-448.

Vasir, J. K., Tambwekar, K., \& Garg, S. (2003). Bioadhesive microspheres as a controlled drug delivery system. International journal of pharmaceutics, 255(1-2), 13-32.

Verma, S., Kaul, M., Rawat, A., Saini, S., \& research. (2011). An overview on buccal drug delivery system. International Journal of Pharmaceutical Sciences Research, 2(6), 1303.

Wang, K., \& Ye, L. (2010). Structure and property of cationic hydroxyethyl cellulose. $J$ Polymer-Plastics Technology and Engineering, 49(8), 807-811.

Woertz, C., Preis, M., Breitkreutz, J., \& Kleinebudde, P. (2013). Assessment of test methods evaluating mucoadhesive polymers and dosage forms: An overview. European Journal of Pharmaceutics and Biopharmaceutics, 85(3), 843-853.

Yadav, V. K., Gupta, A., Kumar, R., Yadav, J. S., \& Kumar, B. (2010). Mucoadhesive polymers: means of improving the mucoadhesive properties of drug delivery system. J. Chem. Pharm. Res, 2(5), 418-432. 